\title{
Outbreak of measles in a non-immunizing population, Alberta 2013
}

\author{
Kershaw $\mathrm{T}^{1^{*}}$, Suttorp $\mathrm{V}^{2}$, Simmonds $\mathrm{K}^{3}$ and St. Jean $\mathrm{T}^{3}$ \\ Canadian Field Epidemiology Program, Public Health Agency of Canada, Edmonton, AB \\ 2 Population and Public Health Department, Alberta Health Services, Lethbridge, AB \\ 3 Surveillance and Assessment Branch, Alberta Health, Edmonton, AB \\ Corresponding author: tanis.kershaw@gov.ab.ca
}

\section{Abstract}

Background: An outbreak of measles was declared in southern Alberta on October 18, 2013, after a case had been reported to the local public health unit in a non-immunized teenager with recent travel to the Netherlands. The teenager had had contact with a large number of unimmunized people while infectious; therefore, the risk of spread was high. The potential for an outbreak of measles in this area had been identified by the lead Medical Officer of Health for South Zone, and planning for an outbreak had begun in August 2013.

Methods: Several public health measures were implemented to control the outbreak: mass immunization clinics; an outbreak dose of measles mumps and rubella (MMR) vaccine for infants 6-12 months old; communication within the affected and surrounding communities; a dedicated measles hotline; a Mobile Measles Assessment Team; and a Measles Assessment Centre.

Results: A total of 42 confirmed cases were identified during the outbreak between October 16 and November 25. Just over half the cases were male (52.4\%). The average age was 12 (range $<1$ to 24 years) and the median age 13 years. There was one hospitalization, and no deaths occurred. All cases were unimmunized. Cases were located in five communities immediately surrounding Lethbridge. All but two cases were epidemiologically linked within 10 households.

Conclusion: The planning that occurred before the outbreak was essential in containing the outbreak to 10 households. To prevent future outbreaks of measles, exploring strategies for increasing immunization coverage rates in unimmunized populations is essential. When immunization acceptance is not uniform, other public health strategies should be planned for and implemented in order to prevent additional spread.

\section{Introduction}

Measles is a highly contagious virus spread through airborne transmission with a greater than $90 \%$ secondary attack rate among susceptible individuals (1). Approximately $30 \%$ of measles cases experience one or more complications, and in developed countries $1-2$ cases per 1,000 will result in death $(1,2)$. Measles is vaccinepreventable: a single dose of measles-containing vaccine is $85 \%-95 \%$ effective, and a second dose raises efficacy to almost $100 \%$ (1). In Alberta, the childhood schedule for a measles vaccine is one dose at 12 months of age and a second dose at 4-6 years of age. The last significant outbreaks of measles in Alberta occurred in 1997 (242 cases), 1999 (17 cases) and 2000 (123 cases).

Health services (including public health services) in Alberta are delivered by Alberta Health Services, which is divided into five zones. The South Zone, the area south of Calgary that includes Lethbridge and Medicine Hat, is composed of diverse cultural groups, many of whom do not support immunization and have historically experienced outbreaks of vaccine-preventable disease. In the County of Lethbridge, the area immediately to the west, north and east of Lethbridge, the last outbreak of measles was in 1997, leaving a large cohort of children born after 1997 not immune to measles disease by natural exposure or immunization. A large demographic group within the County of Lethbridge are families with strong ties to the Netherlands, where, since May 2013, a large-scale outbreak of measles has been occurring in a religious community known to object to immunization 
$(3,4)$. The lead Medical Officer of Health $(\mathrm{MOH})$ for South Zone recognized the risk of measles importation to southern Alberta and began planning for a potential outbreak in August 2013.

This measles preparedness phase used emergency disaster management principles, including use of the incident command system. A number of key strategies and plans were completed for South Zone during this time: 1) implementation of the 2012 National Advisory Committee on Immunization guidelines for measles immunization of health care workers; 2) broad communication to external stakeholders and the public to raise awareness of the risk of measles, promote immunization and educate about measures to minimize transmission for those who do not immunize; 3) engagement between local public health and church leaders, physicians and school administrators; and 4) development of a measles assessment centre plan, triage tools and completion of an inventory and retro-fit of rooms to meet negative pressure standards as per the Canadian Standards Association.

This planning proved to be valuable, because on October 16, 2013, local public health was notified of a suspect measles case in Lethbridge County in a non-immunized teenager with recent travel to the Netherlands. The case had classic measles presentation, with coryza and cough (October 9), followed by fever (October 11) and a maculopapular rash (October 16). Because of information provided to families during the measles planning period, the family was aware of the need to report to public health. This enabled public health officials to obtain laboratory specimens in the client's home on October 16, minimizing the risk of exposure in health care settings. Laboratory results confirmed measles on October 18. The case was known to have interacted, while infected, with a large number of people at a public sporting event, church, school and family events during the Thanksgiving holiday. The majority of these contacts were members of a religious community in which immunization is generally not accepted. On the basis of the risk of spread in a non-immunizing community, an outbreak of measles was declared in South Zone on October 18, 2013.

In this report we present the details of the outbreak, including its epidemiology and the public health measures that were implemented. Lessons learned will be provided for other jurisdictions to consider when dealing with outbreaks of measles, specifically in non-immunizing populations.

\section{Methods}

The outbreak of measles was declared on October 18, 2013, and was confirmed to be over on January 6, 2014. Emergency operations centres were opened in South Zone and at Alberta Health Services and Alberta Health. All three emergency operations centres used principles of the incident command system, which allowed streamlined collaboration and communication among the organizations.

\section{Case finding and data collection activities}

The case definition for measles had been previously established by Alberta Health in the Public Health Notifiable Disease Management Guidelines (5). One clarification was made to the application of the case definition, which was that clinical illness must be evaluated by a health care professional, including public health, and could not be self-reported. Case definitions for confirmed, probable and suspect cases can be seen in Table 1.

Table1. Confirmed, probable and suspect case definitions for measles in Alberta

\begin{tabular}{l|l}
\hline Case classification & Definition \\
\hline Confirmed & A laboratory confirmation of infection in the absence of recent immunization with measles- \\
containing vaccine, meeting one of the following criteria: \\
- Detection of measles virus nucleic acid (e.g. real-time polymerase chain reaction) in a \\
clinical specimen; \\
• Seroconversion or a significant (i.e. fourfold or greater) rise in measles IgG titre between \\
acute and convalescent sera by any standard serologic assay; \\
- Positive serologic test for measles lgM antibody in a person who is either \\
epidemiologically linked to a laboratory-confirmed case or has recently travelled to an \\
area of known measles activity; \\
- Isolation of measles virus from a clinical specimen (e.g. nasopharyngeal swab, urine); or \\
\hline
\end{tabular}




\begin{tabular}{l|l}
\hline Case classification & $\begin{array}{l}\text { Definition } \\
\text { Case. }\end{array}$ \\
\hline Probable & $\begin{array}{l}\text { Clinical illness* in a person who is epidemiologically linked to a laboratory-confirmed } \\
\text { In the absence of both appropriate laboratory tests and an epidemiological link to a } \\
\text { laboratory-confirmed case; or } \\
\text { - In a person who has recently travelled to an area of known measles activity. }\end{array}$ \\
\hline Suspect & $\begin{array}{l}\text { Clinical illness even when the maculopapular rash has been present for less than three } \\
\text { days. }\end{array}$ \\
\hline * Clinical illness is characterized by all of the following features: 1 ) fever $38.3^{\circ} \mathrm{C}$ or greater; 2 ) cough, coryza or conjunctivitis; \\
and 3) generalized maculopapular rash for at least three days.
\end{tabular}

Contact tracing was done with all known contacts of the index case and subsequent cases, but because of the public events the index case had attended there was also broad community exposure. As a result, media releases were issued in South Zone and throughout the province to provide information on measles for members of the public, and a dedicated 'phone line was set up for public inquiries. All suspect cases were investigated by public health and, once confirmed, were reported on a notifiable disease report form and entered into the Communicable Disease and Outbreak Management system.

\section{Epidemiological analysis}

Descriptive epidemiology was completed for the cases on an ongoing basis. Social network analysis was completed on the cases and household contacts of 10 epidemiologically linked families using Pajek software. To complete this analysis, household contact lists were extracted from the case management database for each case and included contacts' immunization history and whether they had a history of disease. In addition, a list of close contacts of cases was extracted to visualize known relationships between cases in the network. The overall and non-immunized household attack rates were calculated for the epidemiologically linked families. Five individuals were excluded from both attack rate calculations: the index case, the co-primary case (probable case), an individual who declined further public health follow-up (case status could not be confirmed) and two individuals who had received two doses of measles-containing vaccine. Twenty-one individuals were excluded from the non-immunized attack rate calculation: 20 who had a self-reported history of disease and one who had had one dose of measles-containing vaccine.

\section{Laboratory methods}

The Alberta Provincial Laboratory performed molecular testing, by real-time polymerase chain reaction (RTPCR), on nasopharyngeal swabs and urine, and conducted serologic testing for measles antibody on blood. Samples testing positive by the measles PCR were referred to the National Microbiology Laboratory in Winnipeg for confirmation and genotyping of the virus. Serologic confirmation was based upon the detection of measles IgM antibody in the acute sample of blood or seroconversion of measles IgG antibody between acute and convalescent samples.

\section{Public health measures}

The outbreak teams reacted quickly and efficiently to implement key public health measures, most of which had been planned during the measles preparedness phase, in order to contain the outbreak of measles. Key measures included the following.

Communication: Timely, transparent, consistent and frequent communication to internal and external stakeholders through traditional and social media was essential during the outbreak. Upon confirmation of the 
index case, the local $\mathrm{MOH}$ immediately met with clergy/pastors and school leaders to inform them and encourage implementation of measures to minimize further spread of disease.

Dedicated measles hotline: A dedicated 'phone line staffed 24/7 during the outbreak was established to address public inquiries. Any 'phone calls about measles received by Health Link, Alberta's telephone service providing free nurse advice and health information to Albertans, were directed to the hotline. The hotline was also available to physicians and served to provide direct triage for the Mobile Measles Assessment Team.

Mobile Measles Assessment Team: The Mobile Measles Assessment Team was established to provide 24/7 assessment of potential cases of measles in the community and divert this population from health care settings. The teams, comprising paramedics and home care nurses in 'phone consultation with Emergency Department physicians, would go to suspected cases' homes to assess individuals for measles, obtain laboratory specimens and provide self-care instructions when indicated.

Measles Assessment Centre: A Measles Assessment Centre was set up outside the Emergency Department of the regional hospital using a Portable Isolation Containment System tent for both assessment and treatment of measles patients. In order to reduce the likelihood of measles transmission in health care settings, and because of the limited availability of negative pressure rooms, the Portable Isolation Containment System tent was a vital public health measure.

Immunization clinics and outbreak doses: Eight public mass immunization clinics were offered in South Zone, and an outbreak dose of MMR was offered to infants 6-12 months of age at well-baby clinics and at the mass clinics.

Quarantine and exclusion: Contacts of cases who were unimmunized were encouraged to self-exclude from school or work until 21 days after the last case was detected in their household. Cases were asked to selfquarantine to their home until they were no longer considered infectious. Quarantine orders were only written upon request, usually for work purposes.

\section{Results}

\section{Descriptive epidemiology}

A total of 43 cases (42 confirmed cases and 1 probable case) were identified during the outbreak. Rash onset for the index case occurred on October 16, and rash onset for the last case occurred on November 25 (Figure 1).

Figure1. Confirmed cases of measles in Alberta by date of rash onset, October 16-November 25, $2013(n=42)$

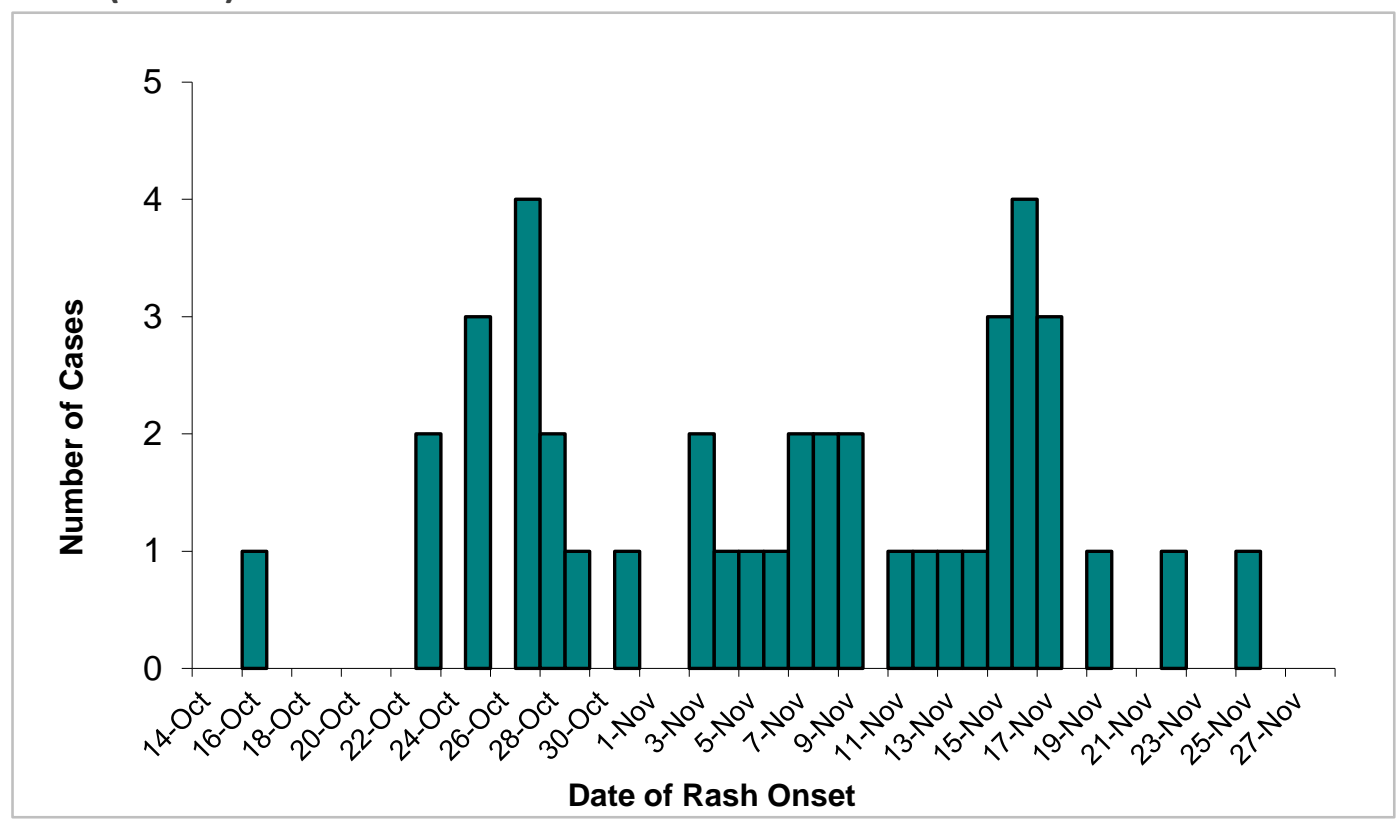


Just over half of the confirmed cases were male (52.4\%). The average age was 12 (range $<1$ to 24 years) and the median age 13 years. Six complications were reported: two cases of pneumonia, three cases of dehydration and one case of otitis media. One of the pneumonia cases required hospitalization. No deaths were associated with this outbreak. Cases were located in five communities immediately surrounding Lethbridge. All cases were unimmunized.

All but two cases were contained to 10 households. These latter two cases had no direct epidemiological link: one resided in a community with measles cases and one resided in a nearby community with no other cases. The relationship between the cases and their household contacts is illustrated in Figure 2. In the diagram, each large black circle represents one household. Seven of the 10 households had direct contact with the index case.

Figure2. Social network diagram of measles cases and household contacts, Alberta, $2013(\mathbf{N}=67)$

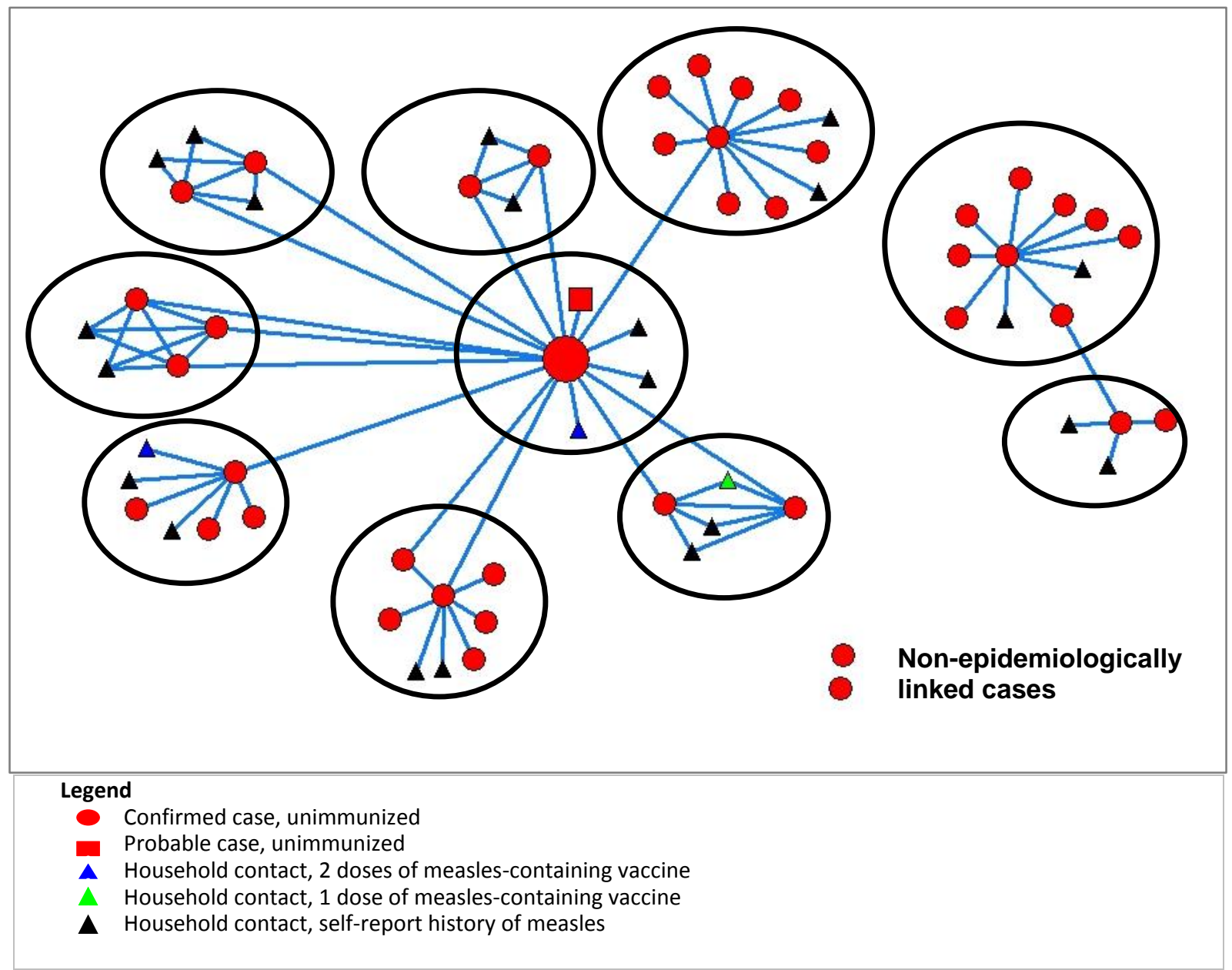

The overall household attack rate was $65 \%$, but the non-immunized household attack rate was $100 \%$. In other words, every person in the 10 households who had not received measles-containing vaccine or did not have a self-reported history of disease acquired measles.

\section{Laboratory results}

Sixteen of the 42 confirmed cases (38\%) were laboratory confirmed. All 16 specimens were sent for genotyping, and 15 were typed. All samples were measles genotype D8 (identical to sequence variant 
MVs/Taunton.GBR/27.12), the same sequence variant identified in the ongoing outbreak in the Netherlands (4) and observed in England in 2013 (6).

Public health measures results

Between October 21 and January 3 public health received a total of 7,857 calls regarding measles. This does not include calls made directly to the local $\mathrm{MOH}$ or to the measles hotline, whose calls were not consistently tracked. The Mobile Measles Assessment Team was mobilized 84 times during the outbreak, and 167 individuals were assessed in the Measles Assessment Centre. A total of 1,302 individuals were immunized at the eight mass clinics.

\section{Discussion}

Imported cases of measles occur in Canada every year, but secondary spread from these cases is usually limited (7). This has generally been the case in Alberta, as this was the first widespread outbreak of measles there since 2000. Because of the direct link of the index case with a large population of susceptible individuals, spread from the index case was expected. However, extensive planning with stakeholders and immediate declaration of an outbreak with confirmation of the index case meant that measles transmission was limited to approximately six weeks. While 42 confirmed cases is still a significant outbreak of measles, it is noteworthy that the cases were contained to 10 households.

There were a few challenges associated with this outbreak. First, public health was aware of a few unreported cases; however, as a result of the strong linkages between public health and the communities, under-reporting of cases was minimal. Second, attack rates for all contacts could not be calculated because of the nature of the public events attended by the index case. Last, given the resources required to manage the outbreak, the data for some public health measures were not consistently tracked, and the data presented are an underestimate.

Despite these challenges, this outbreak of measles was smaller, less severe and shorter in duration than other outbreaks of measles in unimmunized or under-immunized populations seen recently. In 2011, there was a large outbreak of measles in Quebec that lasted almost a year and resulted in 776 cases, with $11 \%$ hospitalized and $8 \%$ suffering complications (8). Of these, $79 \%$ were considered not immune (8). As of February 26, 2014, over 2,600 cases have been reported in the ongoing outbreak in the Netherlands, including 182 hospitalizations and one reported death; $94 \%$ of cases were unimmunized (3). Fraser Health declared an outbreak of measles in a group that opposes immunization for religious reasons on March 8, 2014, and, as of April 8, 2014, had seen 375 cases with two hospitalizations (9). These outbreaks in similar unimmunized populations demonstrate how quickly one measles case can turn into a widespread outbreak and emphasize the importance of preparing in advance and taking immediate action.

This outbreak of measles in Alberta highlights the importance of routine childhood immunization, as all cases in the outbreak were unimmunized. Household contacts who reported a history of disease or one dose of measlescontaining vaccine did not become infected. There are several barriers to achieving high immunization coverage in a population, including low socioeconomic status (10), difficulty in accessing services (10-12) and lack of knowledge or misinformation about immunizations (11-13). In this population, religious convictions and cultural norms were the main barriers. It is important to identify the barriers specific to a community and work with the community to address these barriers. In this outbreak, building a relationship of trust and respect between local public health and the communities led to a collaborative effort to minimize transmission within the broad community.

After the outbreak, work continues in preparation for a future outbreak of measles. A province-wide comprehensive plan is being compiled and includes a number of measures and activities focused on the following: case assessment, diagnosis, infection prevention and control, public health measures and immunization. In addition, a survey has been conducted among the five health zones in Alberta to determine interactions among non-immunizing communities in the province. The information is currently being analyzed and will be used to help predict where vaccine-preventable diseases might spread within the province. 


\section{Conclusion}

This measles outbreak highlights the importance of understanding the demographic nature of local communities, of ongoing surveillance of immunization coverage rates, and of collaboration with both internal and external partners. Recognizing that there are many factors that can contribute to the magnitude and severity of an outbreak, the planning prior to the outbreak and the relationship with the community were key components in containing this outbreak to 10 households. To prevent future outbreaks of measles, exploration of strategies for increasing immunization coverage rates in unimmunized populations is critical. When immunization acceptance is not uniform, other public health strategies should be planned and implemented in order to prevent additional spread of measles, e.g. determining interactions among communities to predict spread, as well as establishing relationships with the communities to better implement infection prevention and control measures should an outbreak occur.

\section{References}

(1) National Advisory Committee on Immunization. Canadian Immunization Guide: Part 4 - Active Vaccines [Internet]. Ottawa: Public Health Agency of Canada; 2012.

http://www.phac-aspc.gc.ca/publicat/cig-gci/p04-meas-roug-eng.php

(2) National Center for Immunization and Respiratory Diseases. Measles. Atlanta: Centers for Disease Control and Prevention; 2009.

http://www.cdc.gov/measles/about/index.html

(3) National Institute for Public Health and the Environment. Mazelenepidemie 2013-2014. The Netherlands: Ministry of Health, Welfare and Sport; 2014 Feb.

http://www.rivm.nl/Onderwerpen/M/Mazelen/Mazelenepidemie_2013_2014

(4) Knol MJ, Urbanus AT, Swart EM, Mollema L, Ruijs WL, van Binnendijk RS, et al. Large ongoing measles outbreak in a religious community in the Netherlands since May 2013. Euro Surveill 2013;18(36).

(5) Alberta Health. Public Health Notifiable Disease Management Guidelines: Measles. Alberta: Government of Alberta; 2013 Nov. http://www.health.alberta.ca/documents/Guidelines-Measles-2013.pdf

(6) Public Health England. Measles cases in England: update to end-June 2013. Health Protection Weekly Report, 2013 Aug 9; 7(32).

(7) Public Health Agency of Canada. Measles. Ottawa, Ontario: Public Health Agency of Canada; 2013 Oct 24. Available from http://www.phac-aspc.gc.ca/im/vpd-mev/measles-rougeole-eng.php

(8) Bureau de surveillance et de vigie. Final report on the provincial outbreak of measles in 2011. Québec: Ministère de la Santé et des Services sociaux; 2012.

http://www.msss.gouv.qc.ca/en/sujets/prob_sante/measles/portrait2011.php

(9) Fraser Health. Health Alert: Measles in Fraser East. Surrey, BC: Fraser Health; 2014. http://www.fraserhealth.ca/your_health/immunizations/measles/news-and-updates/

(10) Santoli J, Szilagyi P, Rodewald L. Barriers to immunization and missed opportunities. Pediatr Ann 1998;27(6):366-374.

(11) Mills E, Jadad A, Ross C, Wilson K. Systematic review of qualitative studies exploring parental beliefs and attitudes toward childhood vaccination identifies common barriers to vaccination. J Clin Epidemiol 2005;58:1081-1088.

(12) Lieber M, Colden F, Colon A. Childhood immunizations: A parental education and incentive program. J Pediatr Health Care 2003;17:240-244.

(13) Evers D. Teaching mothers about childhood immunizations. Am J Matern Child Nurs 2001;26(5):253-6. 


\section{Acknowledgements}

The authors wish to thank Dr. Kevin Fonseca from the Provincial Laboratory for Public Health (ProvLab) for his assistance with writing the laboratory methods section; Martin Lavoie, Dana Paquette and Heather Gagnon for their review of the manuscript; and all the staff from Alberta Health Services, Alberta Health and ProvLab who worked diligently to manage this outbreak of measles.

\section{Conflict of interest}

No conflicts of interest to declare.

\section{Funding}

No external funding was received. 